\title{
Green Foams/Aerogels Based on Nanocellulose derived from Natural Plant Fibers
}

\author{
Wenshuai Chen ${ }^{1,2,}$, , Jun $\mathrm{Cao}^{2}$, Qing Li ${ }^{1}$, Haipeng $\mathrm{Yu}^{1}$, Yixing Liu ${ }^{1}$, Zhichao \\ Quan ${ }^{1}$, Yuanzhu Zhao', Yue Yan ${ }^{1}$, Yanan Zhang ${ }^{1}$, Kuan Cheng ${ }^{1}$, and Jian Li ${ }^{1}$ \\ ${ }^{1}$ College of Material Science and Engineering, Northeast Forestry University, Harbin \\ 150040, P. R. China \\ ${ }^{2}$ College of Mechanical and Electrical Engineering, Northeast Forestry University, Harbin \\ 150040, P. R. China \\ acwsnefu@163.com
}

Keywords: foams; aerogels; nanocellulose; plant fibers

Abstract. Green foams/aerogels based on nanocellulose derived from natural plant fibers have been extensively studied because of their great potential for applications in energy adsorption, energy storage, template, thermal insulation, sensing, and water purification. Progress in fabricating foams/aerogels using nanocellulose as reinforcing fillers or building blocks is reviewed. Firstly, structure and properties of nanocellulose is discussed. Additionally, by means of material classification, various approaches for fabricate foams/aerogels with various types of nanocellulose are introduced. Nanocellulose plays a critical role on the structural, mechanical properties, thermal properties and further functionalizations of foams/aerogels.

\section{Introduction}

Recently, foams/aerogels have attracted considerable attention toward various functional characters, such as lightweight, thermal and acoustic insulation, oil/water separation, catalyst support, sensing, and energy storage. Foams/aerogels are usually synthesized from polymer or carbon-based materials, most of which are derived from petroleum-based products. As science and technology continue to move toward renewable raw materials and more sustainable resources and processes, more attention are recently transferred to fabricate foams/aerogels from renewable resources such as lignocellulosic materials. 


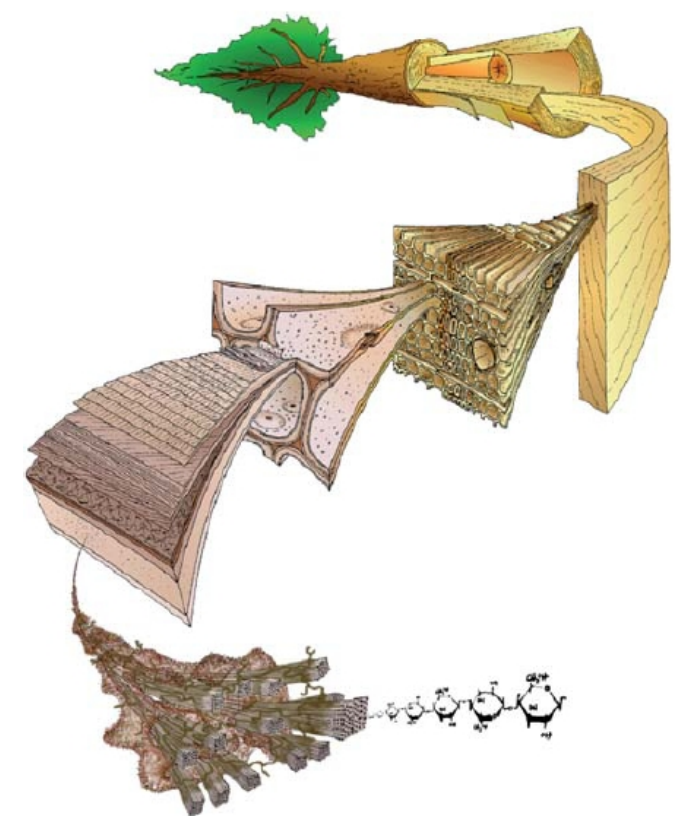

Figure 1 The hierarchical structure of wood from the tree (top left) to the trunk, cells or fibres, cell walls, fibrils and cellulose molecules (bottom right). Art work by Mark Harrington. Copyright University of Canterbury, 1996. ${ }^{[3]}$

In the last 15 years, nanocellulose derived from nature plant fibers, was utilized as reinforcing fillers or building blocks to prepare green foams/aerogel because of its unique structure and properties such as nano-order scale, web-like entangled structure, high Young's modulus, high aspect ratio, and low coefficient of thermal expansion. ${ }^{[1,2]}$ The nanocellulose foams/aerogels display high porosity, advantageous mechanical properties, high thermal stability and good bio-compatibility, which endow them to be used for packaging, thermal insulation, energy adsorption, supercapacitors, and many others.

In this minireview, several demonstrations of nanocellulose foams/aerogels will be illustrated. The preparation of the foams/aerogels with nanocellulose and their characters and applications are also discussed. 

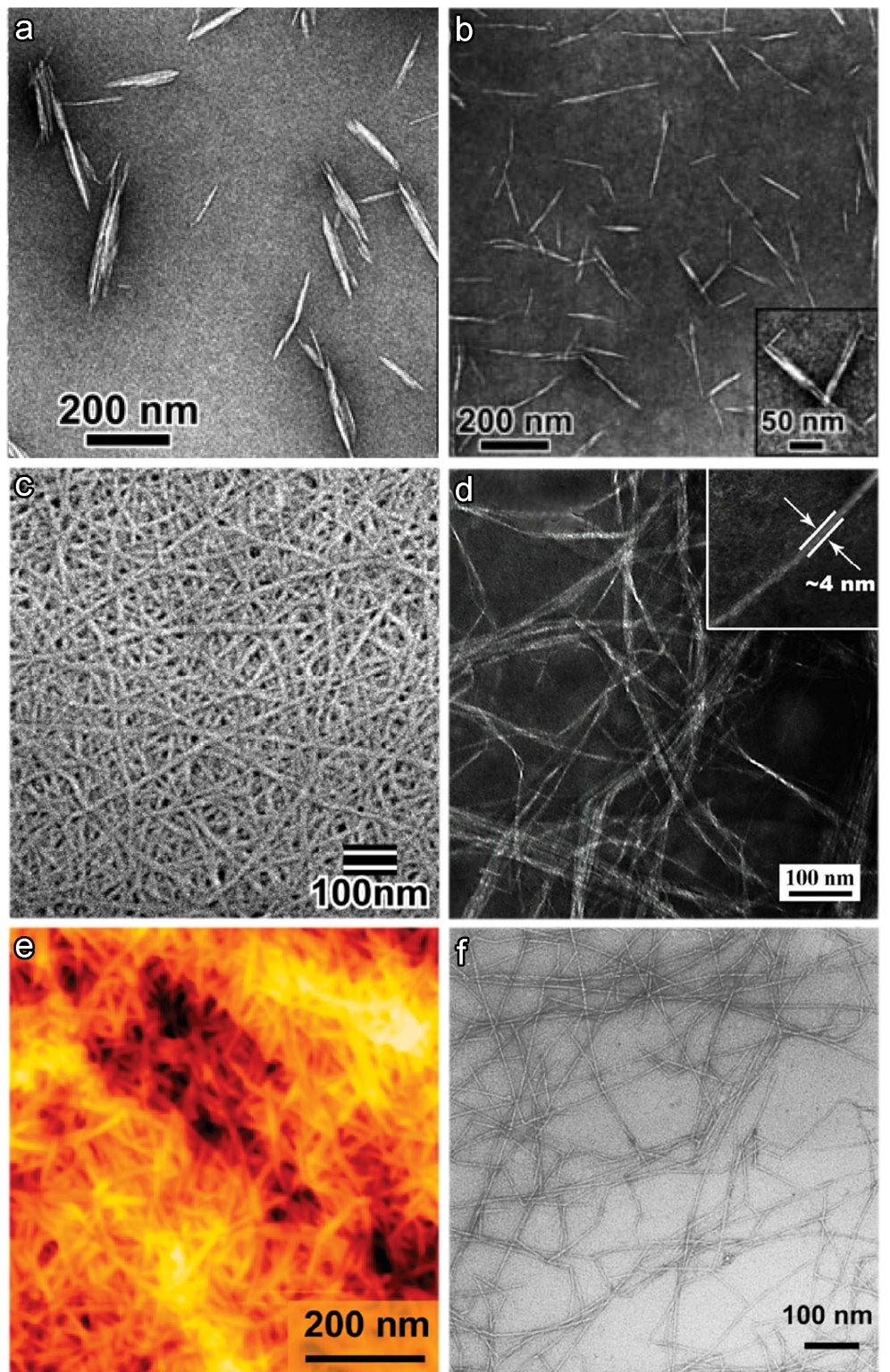

Figure 2 Structure of nanocellulose. (a) TEM micrograph of nanocellulose obtained by sulfuric acid hydrolysis of cotton, ${ }^{[4]}$ (b) TEM micrograph of nanocellulose obtained by sulfuric acid hydrolysis of Avicel, ${ }^{[4]}$ (c) FE-SEM micrograph of wood nanocellulose after one pass through the grinder. ${ }^{[5]}$ (d) TEM micrograph of nanocellulose after treated by high intensity ultrasonication. ${ }^{[6]}(\mathrm{e}, \mathrm{f}) \mathrm{AFM}$ and TEM micrograph of TEMPO-oxidized nanocellulose. ${ }^{[7,8]}$

\section{Structure and properties of nanocellulose}

Nanocellulose can be individualized from many kinds of natural plant fibers such as wood. Trees are recognized as hierarchical nanostructured materials (Figure 1) ${ }^{[3]}$ with strong nanocellulose embedded in hemicellulose and lignin matrix. To produce nanocellulose from wood or other nature plant fibers, chemically pretreatment was usually applied to remove lignin and hemicellulose, resulting in purified cellulose pulps. Next, the cellulose pulps were subjected to nanofibrillation via strong acid hydrolysis, high intensity ultrasonication, TEMPO-mediated oxidation, and mechanical treatment using a high-pressure homogenizer, a grinder, or a high speed blender, resulting in nanocellulose with different structures, shaped and surface chemistry (Figure 2). ${ }^{[4-8]}$ For example, Elazzouzi-Hafraoui et al. fabricated nanocellulose (cellulose nanocrystals) by the sulfuric acid hydrolysis of cellulose from cotton, Avicel, and tunicate. ${ }^{[4]}$ Nanocellulose from cotton and Avicel have a length between 100 and $300 \mathrm{~nm}$ (Figure 2a,b), whereas those from tunicin are several micrometers long and have a whisker-like shape. Abe et al. kept the wood cellulose pulps in water-swollen state after the removal of the matrix, and succeeded in preparing nanocellulose with a uniform width of $15 \mathrm{~nm}$ via nanofibrillation using a 
grinder (Figure 2c). ${ }^{[5]}$ Chen et al. extracted nanocellulose from softwood (from Abies nephrolepis) by using chemical pretreatment combined with high intensity ultrasonication, resulting in nanocellulose with 1-5 nm width and nanocellulose bundles, both of which were entangled into web-like structures (Figure 2d). ${ }^{[6]}$ To prepare homogeneous nanocellulose colloids, Isogai et al. disintegrated never-dried native celluloses after oxidation mediated by the TEMPO followed by a homogenizing mechanical treatment, resulting in individualized, high aspect ratio and slender nanocellulose (Figure $2 \mathrm{e}$ ). ${ }^{[7,8]}$

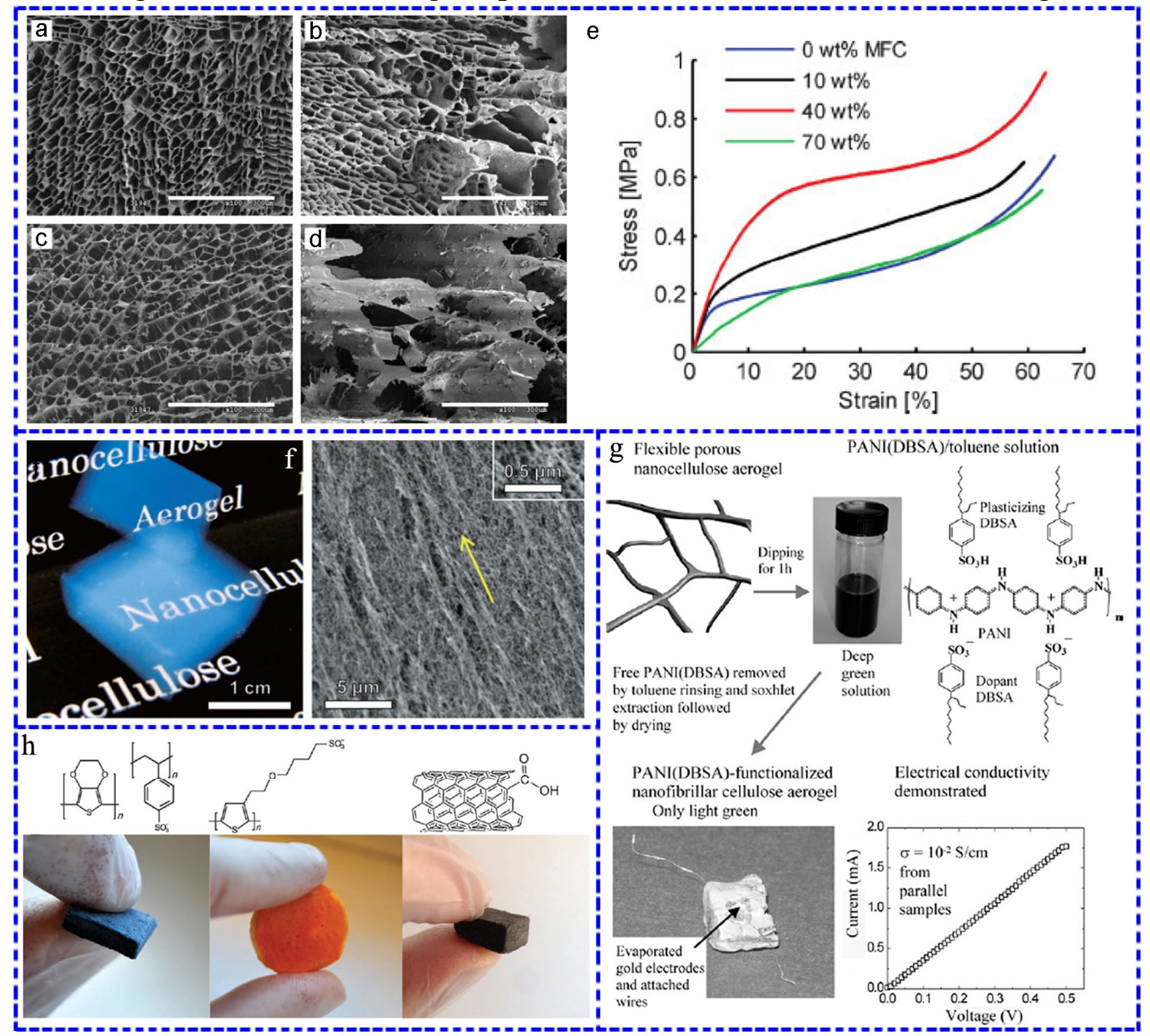

Figure 3 (a-e) The cell structures and compressive stress-strain curves of nanocellulose-reinforced amylopectin foams, ${ }^{[9]}$ (f) Digital photo and SEM image of TEMPO-oxidized nanocellulose aerogel, ${ }^{[10]}$ (g) Functionalization of nanocellulose aerogels using conjugated polymers, ${ }^{[11]}$ (h) ) Optical micrographs of LbL-functionalized nanocellulose aerogels in the dry state, and the corresponding chemical formulas of the functional polyanion used in the LbL. ${ }^{[12]}$

\section{Foams/Aerogels based on nanocellulose}

Utilizing nanocellulose as reinforcing fillers or building blocks, green foams/aerogels with advantageous mechanical properties can be produced (Figure 3) ${ }^{[9-12]}$ Svagan et al. prepared foams using wood nanocellulose reinforced amylopectin-rich potato starch. ${ }^{[9]}$ First, water suspensions were prepared from dissolved starch mixed with nanocellulose. Then, the mixtures were frozen. After removing the water by sublimation, biomimetic foams of high mechanical performance were prepared (Figure 3 a-e). Kobayashi et al fabricated using TEMPO-oxidized nanocellulose as building blocks through acid-induced gelation and supercritical drying. ${ }^{[10]}$ The aerogels are transparent and combine mechanical toughness and good insulation properties (Figure 3f). Pääkkö et al. developed mechanically robust aerogels by freeze-drying of nanocellulose water suspensions. ${ }^{[1]}$ These aerogels can be folded back and forth without fracture. Further, the nanocellulose aerogels can also be functionalized by subsequent treatment. For example, the nanocellulose aerogels were coated with polyanilinedodecylbenzenevsulfonic acid via solvent casting from toluene. The resulting composite aerogels show a moderate conductivity of $\sim 10^{-2} \mathrm{~S} \mathrm{~cm}^{-1}$ (Figure $3 \mathrm{~g}$ ). Hamedi et al. reported a robust and 
rapid method for the layer by layer (LbL) assembly of functional polymers and nanoparticles on crosslinked nanocellulose aerogels with a porosity close to $99 \%$, high strength, and nanoscale shape integrity in water. ${ }^{[12]}$ They assembled conducting polymers, biomolecules, and carbon nanotubes on the nanocellulose aerogels, and enhanced compressive strength, super elasticity in the wet state, fluorescence, elastic mechanoresponsive resistance, and very high charge-storage capacity were observed (Figure 3h). Various types of foams/aerogels based on nanocellulose can be synthesized by utilizing different scientific methods, the foams/aerogels showed multiple functionalities and were utilized in many application areas include energy storage, template, thermal insulation, sensing, and water purification.

\section{Conclusions}

Development of foams/aerogels based on nanocellulosic materials is a rather new but rapidly evolving research area. The present paper introducing the work performed on the fabricating and behavior of foams/aerogels using nanocellulose as reinforcing fillers or building blocks. With the reinforcing of nanocellulose, the foams/aerogels became robust and size stable. The mechanical properties and thermal properties were apparently enhanced. The nanocellulose foams/aerogels can also be functionalized by further treatment. As the intrinsic structure and properties, the nanocellulose foams/aerogels have widely been utilized in many application areas.

\section{Acknowledgements}

This work was supported in part by the Heilongjiang Postdoctoral Foundation (No. LBH-Z14006) and China Postdoctoral Science Foundation (No. 2015T80319).

\section{References}

[1] R. J. Moon, A. Martini, J. Nairn et al: Chemical Society Reviews Vol. 40 (2011), p. 3941

[2] I. Siró and D. Plackett: Cellulose Vol. 17 (2010), p. 459

[3] S.J. Eichhorn: Soft Matter Vol. 7 (2011), p. 303

[4] S. Elazzouzi-Hafraoui, Y. Nishiyama, J. L. Putaux et al: Biomacromolecules Vol. 9 (2007), p. 57

[5] K. Abe, S. Iwamoto and H Yano: Biomacromolecules Vol. 8 (2007), p. 3276

[6] W. Chen, H. Yu, Y. Liu: Carbohydrate polymers Vol. 86 (2011), p. 453

[7] H. Fukuzumi, T. Saito, T. Iwata et al: Biomacromolecules Vol. 10 (2008), p. 162

[8] T. Saito, S. Kimura, Y. Nishiyama, et al: Biomacromolecules Vol. 8 (2007), p. 2485

[9] A. J. Svagan, M. A. S. Samir, L. A. Berglund: Advanced Materials Vol. 20 (2008), p. 1263

[10] Y. Kobayashi, T. Saito, A. Isogai: Angewandte Chemie International Edition Vol. 53 (2014), p. 10394

[11]M. Pääkkö, J. Vapaavuori, R. Silvennoinen et al: Soft Matter Vol. 4 (2008), p. 2492

[12] M. Hamedi, E. Karabulut, A. Marais et al: Angewandte Chemie International Edition Vol. 52 (2013), p. 12038 